IRSTI 27.41 .41

The report is dedicated to the memory of my close friend and classmate D. SC.MD, Professor Smagulov S.S.

\author{
Abylkairov U.U. \\ al-Farabi Kazakh National University, Almaty, Kazakhstan \\ e-mail: U.Abylkairov@gmail.com
}

\title{
The Problem of Single - Determinability Equations of Navier-Stokes
}

\begin{abstract}
The Navier-Stokes equations describing a viscous incompressible fluid have for many decades attracted the attention of scientists working on the problem of solvability of partial differential equations and specialists in the field of numerical analysis due to numerous applications. Despite such interest, the question of the existence and uniqueness of the "on the whole" solution of the non-stationary Navier-Stokes equations in the case of three spatial variables still remains open. S. Smagulov made a great contribution to the development of the theory and numerical methods for solving initial-boundary value problems for the NavierStokes equations. The situation with the numerical solution of these equations is more complex. The fact is that numerical methods that have proven themselves in solving one class of problems are ineffective in solving another class. From the point of view of justification of numerical methods, there is no possibility of using a number of results. The theory of the equation of mathematical physics, since, as mentioned above, they are open to the Navier-Stokes system.

Therefore, the young scientist S. Smagulov of the 1970s of the last century, in order to work successfully in this field of mathematics, combined in himself a specialist in differential equations and also in computational mathematics.
\end{abstract}

Key words: Navier-Stokes equations, regularization, E-approximation, initial-boundary problem.

1.1. Problem statement. The systems of Navier-Stokes, which describes motion of viscous incompressible fluid, have the following form

$$
\begin{gathered}
\overrightarrow{u_{t}}-v \Delta \vec{u}+\nabla p+u_{k} \vec{u}_{x_{k}}=\vec{f} \\
\operatorname{div} \vec{u}=0, \vec{u}(x, 0)=\vec{u}_{0}(x),\left.\vec{u}\right|_{\partial \Omega}=0
\end{gathered}
$$

Here $\vec{u}=\left(u_{1}, u_{2}, u_{3}\right)$-velocity vector, $\mathrm{p}$-function of hydrostatic pressure, and $\vec{f}=\left(f_{1}, f_{2}, f_{3}\right)$-vectorfucntion of sources, $\operatorname{div} u_{0}=0, v$ - viscosity coefficient (value inversely proportional to the Reynolds number), solution is sought in a limited area $\Omega$ with Lipshitz border $\partial \Omega$ three-dimensional space $R^{3}$.

S. Smagulov, already studying at the NSU at the 4th year under the guidance of Ph.D., associate professor B.G. Kuznetsova, began to study methods for the numerical analysis of problems in hydrodynamics. Academician N.N. Yanenko closely followed his scientific growth, posed fundamental problems for solving boundary value problems for the Navier - Stokes system of equations, namely, questions of the approximation of the Navier-Stokes equations by evolutionary type equations and the substantiation of a number of difference methods for solving stationary and nonstationary hydrodynamic 
equations. According to the proposed schemes, numerical calculations were carried out, the results of which were published and transferred to practical application. One of the directions of the study of approximate solutions of the Navier-Stokes equations is based on the use of difference schemes for which the difference energy a priori estimates are valid. In this case, estimates are used for the equations themselves, as well as for some of their $\varepsilon$ approximations.

1.2. The currently known approximations of the Navier-Stokes equations can be mainly divided into two types:

- approximations of the continuity equation (moreover, for the correctness of the approximating problem, it is necessary to make adjustments to the equations of motion)

- approximation (regularization) of only one equation of motion

$\varepsilon$-approximations of the Navier-Stokes equations for a viscous incompressible fluid, which were derived from physical considerations, were proposed in the papers of academician of N.N. Yanenko (by the way the author of the method of fractional steps), B.G. Kuznecov, N.N. Vladimirov [1],[2]. This idea was quickly picked up by prominent French mathematicians Jacques-Louis Lyons, Roger Themes and others. R. Temam [3],[4] proposed a different method of $\varepsilon$-approximation of the Navier-Stokes equations. For these equations, he studied the behavior of the solution at $\varepsilon \rightarrow 0$, a difference scheme was constructed, for which it was shown that, under certain conditions on $\Delta t, \square, \varepsilon$ the solution of the difference problem converges to the solution of the Navier-Stokes equations. An attempt was made in [5] to substantiate difference schemes like fractional steps. We also note other regularizations of the Navier-Stokes equations.

We present an $\varepsilon$-approximation of the NavierStokes equations.

$$
\begin{gathered}
\frac{\partial v^{\varepsilon}}{\partial t}-v \Delta v^{\varepsilon}+v_{k}^{\varepsilon} \frac{\partial v^{\varepsilon}}{\partial x_{k}}+\frac{1}{2} v^{\varepsilon} \operatorname{div} v^{\varepsilon}=f-\nabla p \\
\varepsilon \frac{\partial p^{\varepsilon}}{\partial t}+\varepsilon_{1} p^{\varepsilon}+\operatorname{div} v^{\varepsilon}=0
\end{gathered}
$$

At $\varepsilon_{1}=0$ the convergence of difference schemes for the two-dimensional case is proved under the assumption, that $\Delta t, \square, \varepsilon, \frac{\Delta t}{\varepsilon} \rightarrow 0$ and

$$
\frac{\Delta t}{\sqrt{\varepsilon} \square^{2}} \rightarrow 0
$$

This restrictive condition has been removed by O.A. Ladyzhenskii [6].

We also note other regularizations of the NavierStokes equations, for example, E.G.Dyakonova, D.P.Kaushilayte, V.Ya.Rivkind, A.P.Oskolkov and etc.

Since 1975, a series of papers began to appear, then still a young graduate student THE VC SOAN SSSR S.Smagulov [7],[8],[9]. Two explicit finitedifference schemes are proposed that approximate a quasilinear parabolic system with a small parameter $\varepsilon$, which when $\varepsilon=0$ degenerates into a nonstationary non-linear Navier-Stokes system. The conditions under which the solutions of these finitedifference problems converge to the exact (fairly smooth) solution of the Navier-Stokes equations are clarified. The conditions obtained are less restrictive than the similar conditions given in the papers [6],[10]. The convergence of difference schemes is investigated by the method of energy estimates. S.Smagulov in 1975 proposes an approximation of the Navier-Stokes equations, which is obtained by replacing the continuity equation with another equation. The convergence of the approximate solution that is obtained by solving the replacing system of equations, to solving the Navier-Stokes equations with the speed

$$
\begin{gathered}
\left\|v^{\varepsilon}-v\right\|_{V_{2}^{1}\left(Q_{T}\right)}^{2}=\left\|v^{\varepsilon}-v\right\|_{L^{\infty}\left(0, T ; L^{2}(\Omega)\right)}^{2}+ \\
+\int_{0}^{T}\left\|v^{\varepsilon}-v\right\|_{0}^{2} \underset{W_{2}^{1}(\Omega)}{+} d t \leq c \varepsilon
\end{gathered}
$$

If the solution of the Navier-Stokes equation has the following property

$$
v c_{\Omega}-m>\delta>0
$$

where $\mathrm{m}$ - the exact lower bound of the eigenvalues of the matrix of the strain rate tensor matrix, then the estimate (5) is uniform.

Apparently, for the first time the behavior of a strong solution of problem (1), (2) with $\varepsilon=0, \varepsilon_{1}>$ 0 was considered in the works of S.Smagulov [11], [12] and at the same time in work P.E. Sobolevskii and V.V. Vasilev [13]

Sh. Smagulov introduced the following system:

$$
\begin{gathered}
v_{t}^{\varepsilon}+\left(v^{\varepsilon} \cdot \nabla\right) v^{\varepsilon}+\frac{1}{2} v^{\varepsilon} d i v v^{\varepsilon}= \\
=v \Delta v^{\varepsilon}-\nabla p^{\varepsilon}-\nabla Q^{m}
\end{gathered}
$$




$$
\begin{gathered}
\varepsilon p_{t}^{\varepsilon}+\operatorname{div}^{\varepsilon}=0 \\
\left.v^{\varepsilon}\right|_{t=0}=v_{0}(x),\left.p^{\varepsilon}\right|_{t=0}=p_{0}(x),\left.v^{\varepsilon}\right|_{\gamma_{T}}=0
\end{gathered}
$$

Where $Q_{m}=\sum_{k=1}^{m} \frac{\alpha^{k} p_{k}(x)}{k !}, \alpha(t)-$ a smooth characteristic function on $(0,-\infty), p_{k}(x)=$ $\left.\frac{\partial^{k} p}{\partial t^{k}}\right|_{t=0}-$ is found from the Navier-Stokes equation (unperturbed).

Using the obtained a priori estimates for the higher derivatives and for other structural elements of the problem, we proved the following.

Theorem.

Let $f_{t} \in L^{\infty}\left(0, T ; L^{2}(\Omega)\right), f_{t t} \in L^{2}\left(0, T ; W_{2}^{-1}(\Omega)\right), \gamma \in c^{3}$. Then there is a strong solution to problem (6) - (8) and for this solution the following estimate takes place:

$\left\|v_{t}^{\varepsilon}\right\|_{L^{2}\left(0, T ; W_{2}^{2} \cap \dot{W}_{2}^{1}(\Omega)\right)}+\frac{1}{\varepsilon}\left\|\nabla \operatorname{div} v^{\varepsilon}\right\|_{L^{2}\left(Q_{T}\right)} \leq c<\infty$

By introducing an auxiliary function $Q_{m}(x)$ which provides the conditions for matching at the initial time the solution of the Navier-Stokes equations and the solution of a parabolic system degenerate at $\varepsilon=0$. That is, ensures equality:

$$
\left.\frac{\partial^{k} v^{\varepsilon}}{\partial t^{k}}\right|_{t=0}=\left.\frac{\partial^{k} v}{\partial t^{k}}\right|_{t=0},\left.\frac{\partial^{k}\left(q^{\varepsilon}+Q_{m}\right)}{\partial t^{k}}\right|_{t=0}=\left.\frac{\partial^{k} q}{\partial t^{k}}\right|_{t=0}
$$

Next, we study the internal smoothness of spatial variables.

Sh. Smagulov in 1976-1977, together with Professor Kasimov A.V. the strongest result was obtained on the unambiguous generalized and strong solvability of the diffusion model of an inhomogeneous fluid, which was later called the Kazhikhov-Smagulov equation.

We formulate the initial-boundary problem for this system:

$$
\begin{gathered}
\rho\left[\frac{\partial v}{\partial t}+(v \cdot \nabla)\right]- \\
-\lambda[(\nabla \rho \cdot \nabla) v+(v \cdot \nabla) \nabla \rho]=\mu \Delta v-\nabla p+\rho f \\
\operatorname{div} \vec{v}=0 ; \frac{\partial \rho}{\partial t}+(v \cdot \nabla) \rho=\lambda \Delta \beta
\end{gathered}
$$

Let the mixture move in a limited area

$\Omega \subset R^{3}$ with a fairly smooth border $\mathrm{Y}$ (for example, twice continuously differentiable). For simplicity, let us assume that the boundary $\mathrm{Y}$ is impenetrable and there is no mass transfer between the solution and the external medium:

$$
\left.v\right|_{\mathrm{Y}}=0 ;\left.\frac{\partial \rho}{\partial \vec{n}}\right|_{\mathrm{Y}}=0 ; t \in[0, T]
$$

where $\vec{n}=n_{1}, n_{2}, n_{3}$ - unit vector of external normal Y. Along with the task of mass flow through the boundary Y, another physically reasonable condition can also be considered, when the density values are known on $\mathrm{Y}$ :

$$
\left.\rho\right|_{\mathrm{Y}}=\rho_{\mathrm{Y}}(x, t) ; x \in \gamma ; t \in[0, T]
$$

In addition to the boundary conditions, we supplement the problem with the given by Cauchy:

$$
\left.v\right|_{t=0}=v_{0}(x) ;\left.\rho\right|_{t=0}=\rho_{0}(x) ; x \in \Omega
$$

Regarding $\rho_{0}(x)$ (also $\rho_{\mathrm{Y}}(x, t)$ ) we will assume that this is a positive bounded function:

$$
\begin{gathered}
0<\operatorname{vraimin} \rho_{0}(x)= \\
=m \leq \rho_{0}(x) \leq M=\operatorname{vraimax} \rho_{0}(x)<\infty \\
\Omega \Omega
\end{gathered}
$$

We formulate the main results for this problem obtained by Sh. Smagulov

Theorem 1. Let $v_{0}(x) \epsilon H ; \rho_{0}(x) \in W_{2}^{1}(\Omega), m \leq$ $\rho_{0}(x) \leq M$

$$
\begin{gathered}
f(t)=f(x, t) \in L^{p}\left(0, T ; L^{q}(\Omega)\right), \text { где } \\
p \in[1,2] ; q \epsilon\left[\frac{6}{5}, 2\right] ; \frac{1}{P}+\frac{3}{2 q} \leq \frac{7}{4}
\end{gathered}
$$

Then there is at least one weak solution to problem (9) - (13) if the constants $\mathrm{M}, \mathrm{m}, \mu$ and $\lambda$ satisfy

$$
\lambda \leq 2 \mu(M-m)^{-1}
$$

Theorem 2 .

If $v_{0}(x) \epsilon V ; \rho_{0}(x) \epsilon W_{2}^{1}(\Omega), f(t) \epsilon L^{2}\left(Q_{T}\right) \quad$ and flow is plane-parallel, i.e. $x=\left(x_{1}, x_{2}\right), v=\left(v_{1}, v_{2}\right)$ then, if condition (14) is fulfilled on any finite time interval $(0, T)$, there is a unique strong solution to problem (9) - (13).

And this task is brought by Shaltay Smagulovich to practical results, i.e an approximation of the problem (9) - (13) is proposed, the theorems of convergence, the stability of difference schemes are proved. 
1.3. The important step in the scientific career of Sh.Smagulov is an adoptation of ideas of fictitious area method for the first time for nonlinear equations of mathematical physics, namely for the Navier-Stokes system of equations during 19781979 in papers [14]-[15]. Fictitious areas method (briefly f.a.m.) are pioneers for simple models (Saulev1963 г; lebedev 1964 y, and etc.) reduces the solution of the boundary in regions of complex shape to the solution of boundary-value problems in regions of simple shape (for example, a rectangle or parallelepiped). The reduction is due to the fact that in the domains of simple form, boundary value problems and / or their grid analogues can be solved by efficient, cost-effective methods. There are several fundamentally different approaches for such information. The method of fictitious areas for the nonlinear Navier-Stokes system. Consider a boundary value problem for a nonlinear equation. $\left(u^{\varepsilon} \cdot \nabla\right) u^{\varepsilon}=v \Delta u^{\varepsilon}-\nabla p^{\varepsilon}+f$ в $\Omega_{1}$

$$
\operatorname{div} u^{\varepsilon}=0
$$

$$
\begin{gathered}
\left(u^{\varepsilon} \cdot \nabla\right) u^{\varepsilon}=v \Delta u^{\varepsilon}-\frac{1}{2} u^{\varepsilon} \operatorname{div} u^{\varepsilon}-\frac{1}{\varepsilon} u^{\varepsilon} \text { в } \Omega_{2} \\
\left.u^{\varepsilon}\right|_{\gamma^{+}}=\left.u^{\varepsilon}\right|_{\gamma^{-}},\left.u^{\varepsilon}\right|_{r}=0, \\
v \frac{\partial u^{\varepsilon}}{\partial \vec{n}}-\left.p^{\varepsilon} \delta_{1} \vec{n}\right|_{\gamma^{+}}=\left.v \frac{\partial u^{\varepsilon}}{\partial \vec{n}}\right|_{\gamma^{-}} .
\end{gathered}
$$

The behavior of the solution of problem (15) is investigated as $\varepsilon \rightarrow 0$. An a priori estimate of the solution to the problem (15) was obtained :

$$
v\left\|u_{x}^{\varepsilon}\right\|_{\Omega}^{2}+\frac{1}{\varepsilon}\left\|u^{\varepsilon}\right\|_{\Omega_{2}}^{2} \leq\|f\|_{W_{2}^{-1}(\Omega)}
$$

Further, the existence of at least one generalized solution of problem (15) and its weak convergence in $W_{2}^{1}(\Omega)$ to a generalized solution of the stationary Navier - Stokes equation is proved. The rationale for the method of fictitious domains for the NavierStokes equations and equations for the current function and velocity vortex with inhomogeneous boundary conditions is carried out for the first time. The obtained estimates of the convergence of the solution of the auxiliary problem to the solution of the original problem.

Professor Sh. Smagulov and his students for the first time used the method of fictitious areas to describe incompressible fluid flows in multiply connected areas based on the equations for the current function and velocity vortex, which is a fundamentally new approach to solving the problem of setting boundary conditions for the current function. For the first time carried out the substantiation $\mathrm{mf}$. for problems of flow of a viscous incompressible fluid with inhomogeneous boundary conditions in regions with curvilinear boundaries and complex geometry.

Numerical algorithms based on the method of fictitious areas were developed, which allow studying the flow characteristics of a viscous incompressible fluid around an obstacle in a channel with curvilinear boundaries, characteristics of a convective motion of a viscous incompressible fluid in areas of complex geometry [16-18].

1.4 The next stage of scientific research of Smagulov Shaltay is the problem of the existence of global solutions of the Navier-Stokes equation of compressible continuous media.

The first theorem on the existence of a solution for the Navier-Stokes equations of compressible viscous fluid was obtained by John Nash [19] in 1962, the future winner of the Nobel Prize in economics. He proved the existence theorem for the classical solution of the Cauchy problem "in the small" in time. Somewhat different methods his result was repeated and summarized in the works N. Itay [20], A.I. Wolpert, S.I. Hudyaev [21]

Significant development of non-local theory received in the works A.V. Kozhihov [22-23] and his students V.V. Sheluhin [24], V.A. Vaigant [25] и др.

The first theorems of solvability "on the whole" with respect to time for the model of magnetic gas dynamics were obtained in the work of Sh. Smagulov [26] in the case of a barotropic motion of a viscous gas. In the work of his student A.A.Durmagambetov [27], [28], [29], essential results on the solvability of boundary value problems and the Cauchy problem for degenerate equations of a viscous heat-conducting gas have been obtained. Also, another talented student of Smagulova, Sh. Z.E. Konysbaev, proved the correctness of the initial-boundary value problem for a system of equations of a viscous barotropic gas with an initial density that has degeneracy and takes the magnetic field into account [30-32].

1.4.1 Formulation of the problem. The equations of magnetic gas dynamics in Lagrangian coordinates are:

$$
\frac{\partial v}{\partial t}-\frac{\partial u}{\partial x}=0, v=\frac{\rho^{0}}{\rho}
$$




$$
\begin{gathered}
\rho^{0} \frac{\partial u}{\partial t}=\mu \frac{\partial}{\partial x}\left(\frac{1}{v} \frac{\partial u}{\partial x}\right)-\frac{\partial p}{\partial x}-\mu_{e} H \frac{\partial H}{\partial x} \\
p=k \rho^{0} \frac{\theta}{v} \\
\rho^{0} \frac{\partial \theta}{\partial t}=\lambda \frac{\partial}{\partial x}\left(\frac{1}{v} \frac{\partial \theta}{\partial x}\right)- \\
-p \frac{\partial u}{\partial x}+\frac{\mu}{v}\left(\frac{\partial u}{\partial x}\right)^{2}+\frac{\mu_{e} \mu_{H}}{v}\left(\frac{\partial H}{\partial x}\right)^{2} \\
\frac{\partial}{\partial t}(v \mathrm{H})=\mu_{H} \frac{\partial}{\partial x}\left(\frac{1}{v} \frac{\partial H}{\partial x}\right) .
\end{gathered}
$$

Initially, Cauchy data is known:

$$
\begin{aligned}
& \left.u\right|_{t=0}=u^{0}(x) ;\left.\theta\right|_{t=0}=\theta^{0}(x) ;\left.H\right|_{t=0}= \\
& =H^{0}(x) ;\left.v\right|_{t=0}=1 ; x \in[0,1],
\end{aligned}
$$

where $\left(\rho^{0}, u^{0}, \theta^{0}, H^{0}\right)-$ continuous, $\left(\rho^{0}, \theta\right)$ limited, non-negative and $\rho^{0}$ has degeneration when $\mathrm{x}=1$, i.e. $\rho^{0}(1)=0$

$$
0 \leq \rho^{0}(\mathrm{x}) \leq c<\infty, 0<m_{0} \leq \theta^{0}(\mathrm{x}) \leq M_{0}<\infty
$$

The required functions satisfy the boundary conditions:

$$
\begin{aligned}
\left.u\right|_{x=0} & =\left.u\right|_{x=1}=\left.\frac{\partial \theta}{\partial x}\right|_{x=0}=\left.\frac{\partial \theta}{\partial x}\right|_{x=1}= \\
& =\left.H\right|_{x=0}=\left.H\right|_{x=1}=0
\end{aligned}
$$

and the conditions of approval are met.

Theorem. Let the initial data (18) has the following properties of smootheness:

$$
\begin{gathered}
\left(\rho^{0}(x), u^{0}(x), \theta^{0}(x)\right) \in W_{2}^{1}(\Omega), \\
\frac{p_{x}^{0}(x)}{\left(p^{0}\right)^{\frac{3}{4}}(x)} \epsilon L^{2}(\Omega), \\
\frac{p^{0}(x)}{\inf p^{0}(s)}< \\
c,\left(\left.\sqrt{p^{0}} u_{t}\right|_{t=0},\left.\sqrt{p^{0}} \theta_{t}\right|_{t=0}\right) \epsilon L^{2}(\Omega), H^{0}(x) \epsilon W_{2}^{2}(\Omega) . \\
0 \leq s \leq x
\end{gathered}
$$

Then there is a unique generalized solution to the problem (17)-(19), where $v(x, t)$ strictly positive, bounded function, $\rho^{0}(x) \theta(x, t)$-limited function.

The proof of the theorem is carried out by the method of regularizations. An interesting issue is the study of the correctness of difference schemes of the model of magnetic gas dynamics in Euler variables. In the one-dimensional case, the correctness of difference schemes in Lagrange variables is well studied in the work of Sh. Smagulov and B. Rysbayev [33].

\section{Conclusion}

Scientific works of Sh.S.Smagulova are devoted to the creation and study of efficient algorithms of computational mathematics, the development of numerical methods for the Navier-Stokes equations of great practical and theoretical value, a rigorous mathematical analysis of the solvability of initialboundary value problems for nonlinear equations of composite, non-classical, degenerate types mathematical physics. Sh.S.Smagulov paid much attention to the problems of mathematical modeling and computer technologies in oil production, information technologies for solving practical problems of science and technology.

In the field of computational mathematics, Sh.S.Smagulov made a significant contribution to the development of difference schemes. He constructed and investigated difference schemes for classes of equations:Для стационарных и нестационарных уравнений навье-Стокса

- For heat convection system

- For equations of gas dynamics and magnetic gas dynamics.

Sh. Smagulov is the author of the method of fictitious domains for the non-linear Navier-Stokes equation. For the first time, the method of fictitious regions was applied to describe incompressible fluid flows in one or multiply connected domains, and the method of fictitious domains with non-uniform boundary in regions with curvilinear boundaries was substantiated.

Developed numerical algorithms based on the method of fictitious areas.

- Sh. Smagulov made a great contribution to the development of the theory of solvability of the non-linear Navier-Stokes equations, the equations of thermal convection, magnetic gas dynamicsi

- The correctness of the problems of degenerate equations of magnetic gas dynamics 
- The correctness of the problems of degenerate equations of a reacting mixture of gases

Solvability and convergence of the $\varepsilon$ approximation of the Navier-Stokes equations, the temperature model of homogeneous and inhomogeneous liquids; and taking into account the dissipation of energy.

\section{References}

1. N.N. Yanenko. The method of fractional steps for solving multidimensional problems of mathematical physics, Novosibirsk, Science (1967): 225.

2. N.N. Vladimirova, B.G. Kuznecov, N.N. Yanenko. Numerical calculation of symmetric flow of a plate around a plane flow of a viscous incompressible fluid. Some questions of computational and applied mathematics. Novosibirsk (1966): 29-35.

3. Temam R. "Une method d'approximation dela solution des-equations de Navier-Stokes." Bull.Soc.Math.France 96 (1968): 115-152.

4-5. Temam R. Sur l'approximation de la solution des equations de Navier-Stokes parla method de pas fractionnaries (I)(II),Arzhive Rat.mech.Anal.,32.

6. O.A. Ladyzhenskaya. Mathematical problems of the dynamics of a viscous incompressible fluid. Science (1970): 288.

7. A. Smagulova. "On a non-linear equation approximating the Navier-Stokes equation." Proceedings of the Vth All-Union Seminar on Numerical Methods of Viscous Fluid Mechanics Novosibirsk (1975): 123-134.

8. B.G. Kuznecov, Sh. Smagulov. "On difference schemes with a small parameter approximating the Navier-Stokes equations." Proceedings of the Vth All-Union Seminar on Numerical Methods of Viscous Fluid Mechanics, Novosibirsk (1975): 109-122.

9. B.G. Kuznecov, Sh. Smagulov. Approximation of the hydrodynamic equation. " Numerical Methods of Continuum Mechanics" no. 2, (1975): 158-175.

10. A.P. Oskolkov. "On some convergent difference schemes for the Navier-Stokes equations." Proceedings of the Steklov mathematical Institute 125 (1973): 164-172.

11. B.G. Kuznecov, Sh. Smagulov. "On the rate of convergence of solutions of a single system of equations with a small parameter to the solution of the Navier-Stokes equations." Mathematical models of fluid flow. ITAM SB AS USSR Novosibirsk (1978): 158-175.

12. Sh. Smagulov. "On parabolic approximation of the Navier-Stokes equations." Numerical Methods of Continuum Mechanics, VTS and ITPM of Soan USSR vol. 10, no. 1979: 137-149.

13. P.E. Sobolevskii, V.V. Vasilev. On one Eapproximation of the Navier-Stokes equations. "Numerical Methods of Continuum Mechanics" vol. 9, no. 5 (1978): 115-139.

14. A.N. Bugrov, Sh. Smagulov. "The method of fictitious domains in boundary value problems for the Navier-Stokes equations." Mathematical models of fluid flow (1978): 79-90.

15. Sh. Smagulov. "The method of fictitious domains for boundary value problems of the NavierStokes equations." VTS SO AN USSR. Preprint. 68: 22.

16. A.V. Kazhihov, Sh. Smagulov. "On the correctness of boundary problems of a diffusion model of an inhomogeneous fluid." DAN SSSR vol. 234, no. 2 (1377): 330-332.

17. Sh. Smagulov. "Approximation of the equations of one model of an inhomogeneous fluid." Numerical Methods of Continuum Mechanics vol. 8, no. 2 (1977): 112-124.

18. Sh. Smagulov. "On a variant of approximation of the Navier-Stokes equations." Partial differential equations, Novosibirsk (1980): 57-62.

19. J. Nesh. "The problem de Cauchy pour les equation diffeentielles d'un fluide general." Bull.Soc.Math.France vol. 90, no.4 (1962): 487-497.

20. Itaya N. "The existence and uniqueness of the solution of the equations describing compressible viscous fluid flow." proc. Jap. Acad. vol. 46, no. 4 (1970): 379-382.

21. Wolpert A.I., Hudlev S.I. "On the Cauchy problem for composite systems of nonlinear differential equations." Mathematical collection vol.87, no.4 (1972): 504-528.

22. Kazhihov A.V. "The correctness "on the whole" of mixed boundary value problems for a model system of equations of a viscous gas." Dynamics of continuous media no. 21(1975): 18-47.

23. Kajihov A.V. On the Cauchy problem for viscous gas equations." Siberian mathematical journal vol. 23, no.1 (1982): 60-64.

24. Kajihov A.V., Sheluhin V.V. "Unambiguous solvability of the initial-boundary value problems for the one-dimensional viscous gas equations." Applied mathematics and mechanics vol. 41, no. 2 (1977): 282-291. 
25. Vaigant V.A., Kazhihov A.V. "On the existence of global solutions of the two-dimensional Navier-Stokes equations compressible viscous fluid" Siberian mathematical journal vol. 36, no.6 (1995): 1283-1316.

26. Smagulov Sh. "On the correctness of some problems for the equations of magnetic hydrodynamics." Mate.models of fluid flows. Novosibirsk : In-t Theor. And PM .mechanics of USSR Academy of Sciences (1978): 257-266.

27. Durmaganbetov A.A. Solvability of some nonlinear problems of gas dynamics: Thesis of candidate of Phys.-math. sciences': 01.01.02. Almaty (1987): 75.

28. Smagulov Sh., Durmaganbetov A.A., Iskenderova D.A. "The Cauchy problem for the equations of magnetic gas dynamics." Differential equations vol. 29, no.2 (1993): 337-348.

29. Smagulov Sh., Iskenderova D.A. The Cauchy problem for the equations of a viscous heatconducting gas with a degenerate density // Journal of Computational Mathematics and Mathematical Physics. - 1993. - T. 33, no. 8. - C. 12511259.

30. Smagulov Sh., Konysbaev Zh.E. "On the correctness of the initial-boundary value problem for the equations of a viscous heat-conducting gas with a degenerate density." Continuum dynamics vol. 93 (1989):119-130.

31. Smagulov Sh. Iskenderova J.A. "The Caushy problem for the equations of a viscous heat - conducting gas with degenerate density." Comput.math.and math.phys vol 29, no.8 (1993): 1109-1117.

32. Smagulov Sh., Ourmaganbetov A.A., Iskenderova J.A. "The Caushy problem for the equations of magnetic-gas dynamics." Different. Equations vol. 29, no. 2 (1993): 278-288.

33. Smagulov Sh., Rysbaev B.R. "On convergent difference schemes for viscous gas equations." Doc.USSR ACADEMY OF SCIENCES vol. 287 , no. 3 (1988): 558-559. 